\title{
Production and 3D printing processing of bio-based thermoplastic filament
}

\author{
Eleni Gkartzou, Elias P. Koumoulos, and Costas A. Charitidis* \\ Research Unit of Advanced, Composite, Nano Materials \& Nanotechnology, National Technical University of Athens, \\ School of Chemical Engineering, 9 Heroon Polytechniou St., Zographos, Athens 15780, Greece
}

Received 22 July 2016 / Accepted 22 October 2016

\begin{abstract}
In this work, an extrusion-based 3D printing technique was employed for processing of biobased blends of Poly(Lactic Acid) (PLA) with low-cost kraft lignin. In Fused Filament Fabrication (FFF) 3D printing process, objects are built in a layer-by-layer fashion by melting, extruding and selectively depositing thermoplastic fibers on a platform. These fibers are used as building blocks for more complex structures with defined microarchitecture, in an automated, cost-effective process, with minimum material waste. A sustainable material consisting of lignin biopolymer blended with poly(lactic acid) was examined for its physical properties and for its melt processability during the FFF process. Samples with different PLA/lignin weight ratios were prepared and their mechanical (tensile testing), thermal (Differential Scanning Calorimetry analysis) and morphological (optical and scanning electron microscopy, SEM) properties were studied. The composition with optimum properties was selected for the production of 3D-printing filament. Three process parameters, which contribute to shear rate and stress imposed on the melt, were examined: extrusion temperature, printing speed and fiber's width varied and their effect on extrudates' morphology was evaluated. The mechanical properties of $3 \mathrm{D}$ printed specimens were assessed with tensile testing and SEM fractography.
\end{abstract}

Key words: 3D Printing processability assessment, Fused filament fabrication, Additive manufacturing, Biobased 3D printing filament

\section{Introduction}

In the last decade, issues concerning environmental pollution and the increasing awareness of limited resources, have motivated the scientific community to study and optimize renewable alternatives to traditional petroleum-derived plastics, like biobased composite materials that are sourced from carbon-neutral feedstocks [1]. Lignin is a highly aromatic biopolymer, abundantly found in the fibrous part of various plants and extracted as a byproduct of wood pulping industries. Kraft lignin (sulfate lignin) is isolated in the so-called delignification process, from black liquor by precipitation and neutralization with an acid solution $(\mathrm{pH}=1-2)$, and subsequently dried to a solid form [2]. It is estimated that only $2 \%$ of the industrially extracted lignin is exploited for low-volume, niche applications, while the rest is often relegated to a low efficiency energy recovery via combustion or as a natural component of animal feeds [3]. Thus, the development of ways to convert lignin to new high-value products is an active area of research,

*e-mail: charitidis@chemeng.ntua.gr dealing with the main drawbacks of lignin usage regarding the low-purity standards, heterogeneity, smell and color problems of the existing commercial lignins. It is recognized that blending lignin with polymers is a convenient and inexpensive method to create new materials with tailored properties, such as hydrophobicity, stiffness, crystallinity, thermal stability, Ultraviolet (UV) blocking ability and to reduce the overall cost of the material $[4,5]$.

The ecosystem of $3 \mathrm{D}$ printing plastic market consists of numerous research and development activities and is projected to reach USD 692.2 Million by 2020, at a Compound Annual Growth Rate (CAGR) of $25.7 \%$ from 2015 to 2020 [6]. Among the various commercially available specialty filaments for Fused Filament Fabrication (FFF) processing, materials mimicking wood texture and properties are a separate category, because of their ability to create objects with the tactile feel of wood without any need for specialized woodworking tools. Furthermore, they require less maintenance and preservatives, since they are more resistant to organic decomposition, while maintaining their biodegradability. PLA/Lignin (poly(lactic acid)/Lignin) 3D printing filaments are an alternative option 
for lignin exploitation and filament cost reduction and can be used in rapid prototyping, presentation models and consumer products, among others. Poly(lactic acid) is a biodegradable thermoplastic, which is produced via fermentation or chemical synthesis from a bio-derived monomer, lactic acid (2-hydroxy propionic acid) [7]. The carbon in PLA originates from atmospheric carbon dioxide, which is immobilized in glucose by photosynthesis; therefore, its impact on the environment during production and disposal (carbon footprint) is low compared to other petro-based polymers. PLA is widely used in 3D printing applications, since it is one of the most user-friendly materials that can be easily processed with FFF, without emitting toxic fumes. However, its low thermal stability, high degradation rate during processing and brittle behavior have to be addressed. It has been suggested that the presence of lignin increases thermal stability and flammability under oxidative and nonoxidative conditions, due to the formation of char, which acts as a protective layer preventing oxygen diffusion [8].

Extrusion-based 3D printing techniques use temperature as a way of controlling the material state, for the successful extrusion and deposition of semi-molten thermoplastic fibers, on a flat surface. In a typical FFF process, a filament feedstock is supplied to the system by an electric motor-controlled pinch roller mechanism $[9,10]$. Material is liquefied inside a reservoir, contained in a heated metal block with a machined channel, so that it can flow through the print head's nozzle and fuse with adjacent material before solidifying. This approach is similar to conventional polymer extrusion processes, except the extruder is vertically mounted on a plotting system (print head) rather than remaining in a fixed horizontal position [10]. After its deposition, the solidifying material is referred to as a fiber or road. The part is produced by superimposing a specified number of layers, where each of them is generated by a specific pattern of fibers. The formation of bonds among individual fibers in the FFF process consists of complicated heat and mass transfer phenomena coupled with thermal and mechanical stress accumulation and phase changes. The strength of these bonds depends on the growth of the neck formed between adjacent fibers and on the molecular diffusion and randomization at the interface [11]. As a natural consequence of this manufacturing approach, the part's internal microstructure consists of fibers with partial bonding among them and voids [12] and can be assimilated to a composite two-phase material with inherently orthotropic properties [13]. Individual fibers are significantly stronger in the axial direction and resemble the fibers in a composite; however, the structure shows weaker behavior in the direction where stresses need to be carried through fiber-to-fiber or layer-tolayer adhesion [12].

Three dimensional printers employing the FFF technology are Computer Numerical Control (CNC) machines, whose function is defined by a program containing coded alphanumeric data (G-code). G-code sets of commands are typically generated by Computer-Aided Manufacturing (CAM) software, which uses topological information from 3D Computer-Aided Design (CAD) data along with user-defined processing and toolpath parameters, to create virtual slices of the object to be manufactured and to calculate the toolpath and the material's Volumetric Flow Rate (VFR), in order to form the successive cross sections of the physical part. In most CAM programs for lower end FFF 3D printers, VFR is a function of the linear feed velocity of the filament and of several design parameters related to the toolpath (e.g. the width and height of individual fibers, defined by extrusion width and layer height parameters) [14]. The pressure-driven mass flow of the non-Newtonian polymeric melt through the nozzle is mainly related to nozzle geometry, pressure drop and melt's apparent viscosity. This flow can be described as a fully developed, laminar flow through a capillary die with a circular cross section $[14,15]$. The necessary pressure for fiber extrusion is applied by the solid portion of the 3D printing filament which acts as a piston, as it is pushed by a pinch-roller feed mechanism into a melting reservoir, placed on the upper part of the nozzle. Volumetric flow rate along with extrusion temperature are two material-dependent factors which contribute on the shear rate and stresses imposed on the melt during extrusion. In the case of composite $3 \mathrm{D}$ printing materials, these factors are significantly influenced and limited by the filler's dispersion and agglomeration [16].

This study is divided in two main sections; the first section involves the preparation and characterization of bulk samples of the composite material with increasing lignin content and $3 \mathrm{D}$ printing filament production. The second section concerns the selection of suitable toolpath and process parameters based on the produced filament's response during FFF processing, focusing on extrusion temperature, print head's velocity and extrusion width. By understanding the relationship between processing conditions and physical phenomena involved in the material's extrusion and deposition, suitable bounds for these parameters were derived. The optimum velocity and temperature values were used for the fabrication of tensile specimens with $100 \%$ nominal density and three different extrusion widths, produced by three brass nozzles with different diameter $(0.2,0.3$ and $0.4 \mathrm{~mm})$, in order to measure the tensile properties of the finished parts and to compare them with the properties of the bulk material. Pure PLA filament was produced and processed under the same conditions, to be used for comparison. Fractographic analysis of tensile failure was carried out with Scanning Electron Microscopy (SEM). Also, a qualitative assessment of the filler's dispersion and agglomeration into the polymeric matrix, as well as its effect on surface morphology and diameter of individual fibers was made with optical microscopy.

\section{Experimental details}

\subsection{Materials}

Commercial PLA pellets under the grade name "INGEO 2003D" were supplied by Natureworks LLC, with number average molecular weight $M_{\mathrm{n}}(\mathrm{g} / \mathrm{mol})=114.317$, weight average molecular weight $M_{\mathrm{w}}(\mathrm{g} / \mathrm{mol})=181.744$ and $4.3 \mathrm{wt} . \%$ D-isomer content [17]. A purified form of kraft pine lignin (Indulin AT) was supplied by MWV Specialty Chemicals, in the form of free-flowing powder with a wide distribution of particle diameter, as depicted in the SEM micrographs of Figure 1. Both materials were used without chemical treatment 
(a)

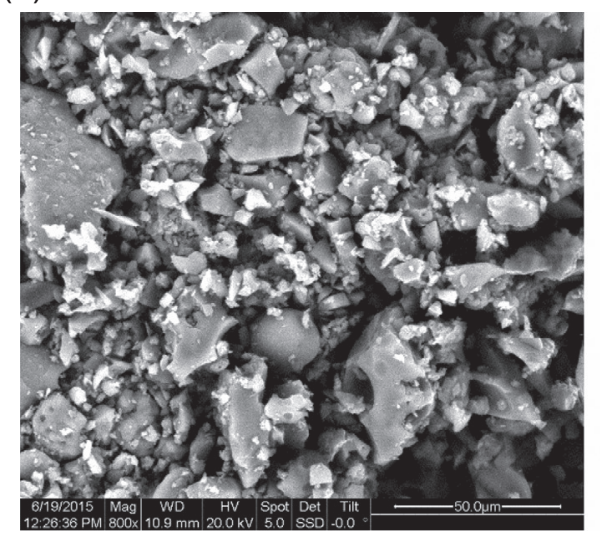

(b)

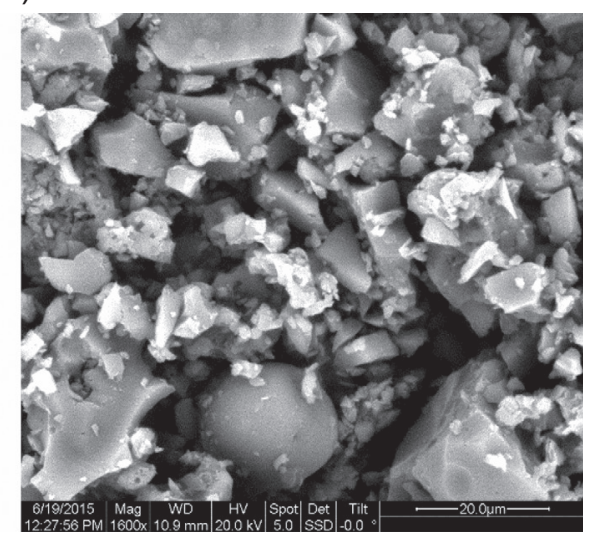

Figure 1. SEM micrographs of kraft lignin (Indulin AT) particles (a) $\times 800$ and (b) $\times 1.600$ magnification.

for the preparation of the blends. Prior to processing, the components were vacuum-dried at $50{ }^{\circ} \mathrm{C}$ for $24 \mathrm{~h}$ and weighted in a high precision scale.

\subsection{Blending}

Firstly, $40 \mathrm{~g}$ samples of PLA/Lignin blends with different lignin concentrations $(5,10,15,20 \%$ percentage by weight on the dry polymer - Table 1) were prepared by melt mixing in a twin-screw Brabender internal mixer. Mixing time for each sample varied from 10 to $13 \mathrm{~min}$, at $35 \mathrm{rpm}$ rotating speed of the screws and mixing temperature between $180-190{ }^{\circ} \mathrm{C}$ (depending on lignin content). To remove residual stresses and air bubbles caused by the blending process, the semi-molten material was collected from the mixing chamber and placed in an aluminum mold, which was inserted in a Dake thermopress to form a $15 \times 60 \times 1 \mathrm{~mm}$ plate. The plates of the thermopress were heated at $120{ }^{\circ} \mathrm{C}$ and heating was switched off when maximum load was applied on the mold. All samples used for the bulk material's characterization were cut from the aforementioned plates and kept under room temperature in a glass desiccator, to prevent moisture absorption.

\subsection{Characterization}

The effect of increasing lignin content on the bulk material's thermal and mechanical properties was evaluated with Differential Scanning Calorimetry (DSC) and tensile testing. The phase morphology of the samples was examined with an Axio Imager A2m optical microscope and AxioCam ICc5 CCD camera (Carl Zeiss, Oberkochen, Germany) and SEM micrographs were taken with Nova NanoSEM 230 scanning electron microscope (FEI Company) with an acceleration voltage of $5 \mathrm{kV}$. Samples for optical inspection were cut with a rotating saw and embedded in cold mounting epoxy resin. The embedded samples' surface was grinded with fine silicon carbide abrasives to remove defects introduced by sectioning. Tensile specimens were directly cut from the thermopressed plates of the compounded material with a dumbbell-shaped specimen cutting die, with a $18 \times 3 \times 1 \mathrm{~mm}$ reduced gage section. Measurements of mechanical properties of specimens
Table 1. Sample composition.

\begin{tabular}{lcc}
\hline Sample & PLA wt.\% & Lignin wt.\% \\
\hline LPLA00 & 100 & 0 \\
LPLA05 & 95 & 5 \\
LPLA10 & 90 & 10 \\
LPLA15 & 85 & 15 \\
LPLA20 & 80 & 20 \\
\hline
\end{tabular}

were performed at room temperature with a Zwick tensile tester, model 1120 equipped with a $2000 \mathrm{~N}$ load cell. Both Young's modulus and elongation measurements were made at a constant crosshead speed of $2 \mathrm{~mm} / \mathrm{min}$. Each value of mechanical properties reported is an average of five specimens. DSC analyses were performed with DSC Q200 TA Instruments (New Castle, DE, USA). The thermal history of samples was erased by a preliminary heating cycle, followed by a cooling cycle from 200 to $0{ }^{\circ} \mathrm{C}$ and a second heating cycle from 0 to $200{ }^{\circ} \mathrm{C}$. Both cooling and heating rates were set at $10{ }^{\circ} \mathrm{C} /$ min. The samples' mass ranged from 6.90 to $8.36 \mathrm{mg}$ and they were encapsulated in aluminum pans. An empty pan was used as reference. The glass transition temperature $\left(T_{\mathrm{g}}\right)$ cold crystallization temperature $\left(T_{\mathrm{cc}}\right)$, double melting peak temperatures $\left(T_{\mathrm{m} 1,2}\right)$, cold crystallization enthalpy $\left(\Delta H_{\mathrm{cc}}\right)$ and melting enthalpy $\left(\Delta H_{\mathrm{m}}\right)$ were determined from heating scans. Thermogravimetric Analysis (TGA) of softwood kraft lignin was carried out based on global mass loss with a Netzsch 409 EP analyzer, from which lignin's decomposition pattern can be derived. The analysis was conducted under nitrogen atmosphere with a heating rate of $10{ }^{\circ} \mathrm{C} / \mathrm{min}$. The characterization of $3 \mathrm{D}$ printed fibers and tensile specimens involved optical microscopy and tensile testing. Brightfield illumination was used to observe the surface roughness of PLA/Lignin fibers. Also, by exploiting PLA's transparency and alterations in the incident light's state of polarization during its interaction with lignin, a qualitative evaluation of the dispersion of lignin's agglomerates in the fibers' bulk volume was conducted. AxioVisio digital image processing software was used to measure the diameter of the fibers from the micrographs captured by the CCD camera. Since no special standard exists for the characterization of FFF parts, tensile specimens based on a scaled 
down version of ASTM D638 Type I with a $36 \times 8 \times 2 \mathrm{~mm}$ gauge section, were 3D printed using Zmorph $2.0 \mathrm{~S}$, a commercial FFF Cartesian XZ system (Zmorph LLC, Wroclaw, Poland). Tensile testing was carried out under the same conditions as the bulk material specimens, with the same crosshead speed of $2 \mathrm{~mm} / \mathrm{min}$. Both pure PLA and composite PLA/ Lignin filaments were used as raw materials. The 3D printed specimens had $100 \%$ infill density in order to make a comparison with the tensile properties of the bulk material and to eliminate errors from G-code generation. Divergence from the nominal dimensions of the tensile specimens introduced by the fabrication process, was measured for each specimen with a high precision digital caliper and the average of 10 measured values for each dimension was used for stress calculations. Fractographic analysis of tensile failure was carried out with scanning electron microscopy. The fractured samples were sputter-coated with a thin layer of gold before observation.

\subsection{Production of 3D printing filament}

The 3D printing filament needs to be able to provide and sustain the pressure needed to drive the extrusion process. Failure to do this results in filament buckling, which occurs when the extrusion pressure is higher than the critical buckling load that the filament can support $[10,14,18]$. The filament's elastic modulus determines its load carrying ability and melt viscosity determines the resistance to extrusion (or extrusion pressure). As a result, the composition with 5 wt.\% lignin content was selected for the production of 3D printing filament with $1.75 \mathrm{~mm}$ nominal diameter, which is compatible with the 3D printer's feeding system. Subsequently, a Boston-Mathews single-screw extruder with an L:D ratio of $25: 1$ with a $1.8 \mathrm{~mm}$ diameter extrusion die was used to obtain PLA/Lignin filament and several combinations of process parameters were tested in order to achieve enhanced filler dispersion and constant filament diameter. A processing temperature profile of 185-195-205-205-195 ${ }^{\circ} \mathrm{C}$ from feed section to die was selected along with a fixed screw speed at $16 \mathrm{rpm}$. The extrudate was collected by a conveyor belt equipped with cooling fans, whose speed was matched to the extrusion speed in order to control the diameter of the filament. The composite filament and pure PLA filament (processed under a 180-190-200-200-190 ${ }^{\circ} \mathrm{C}$ temperature profile) were used to obtain 3D printed specimens and individual fibers. The produced filament was conditioned in room temperature inside sealed plastic bags, in the presence of silica gel, to avoid moisture absorption. Filament sections with tight tolerances were selected for specimen fabrication. Each section's diameter was measured every $1 \mathrm{~cm}$ with a high precision digital caliper and average diameter and standard deviation were calculated. From Figure 2 it can be seen that the measured filament diameters are normally distributed around the mean value, with $0.02 \mathrm{~mm}$ standard deviation, meaning that $95 \%$ of the filament used has a \pm 0.04 tolerance. A tight diameter tolerance is important during the material melting and deposition process. The heating of the filament inside the print

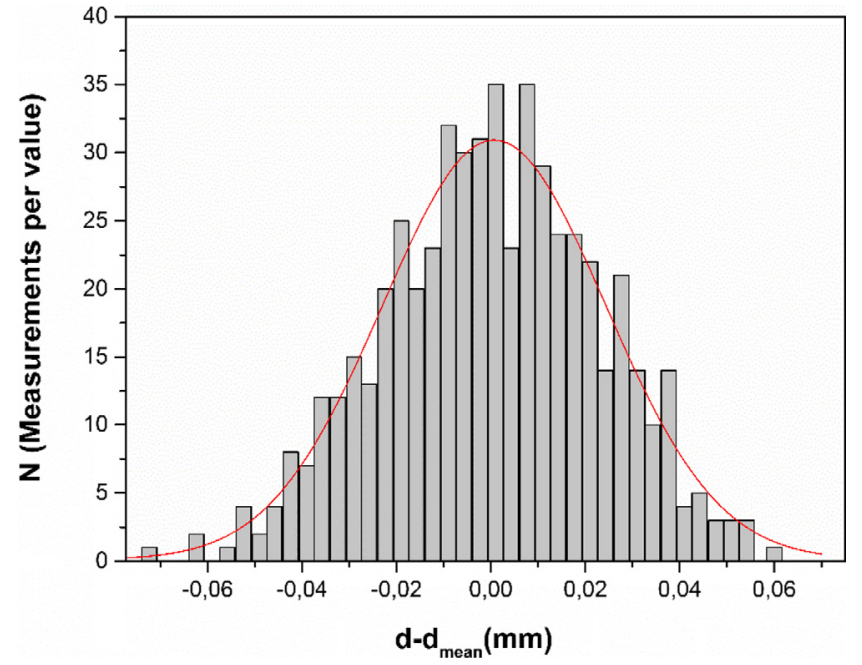

Figure 2. Diameter distribution of filament sections used for specimen fabrication.
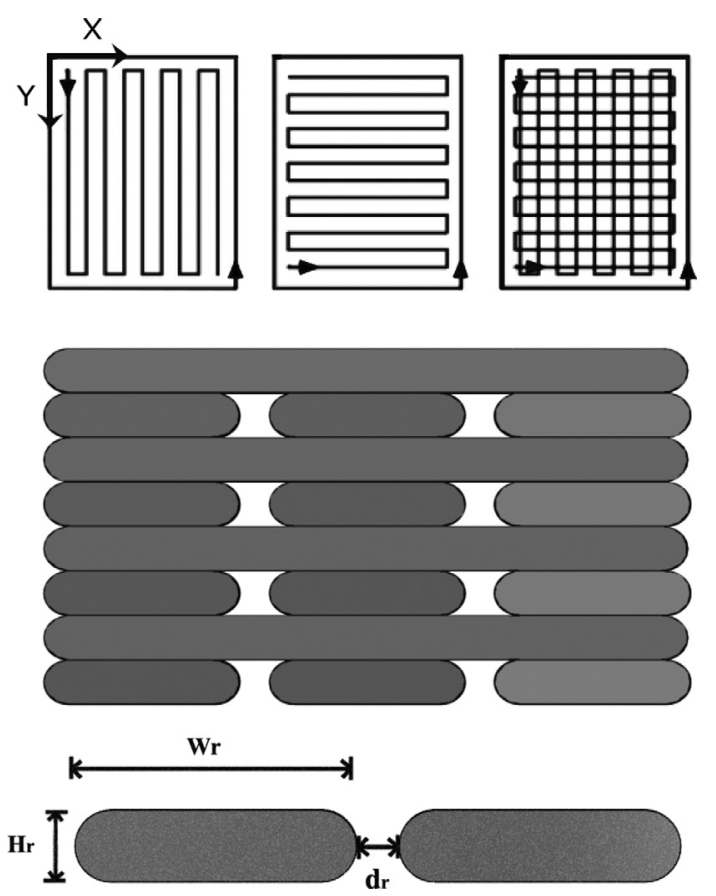

Figure 3. Illustrations of toolpath for rectilinear infill pattern with $90^{\circ}$ raster angle generation and cross sections of the produced pattern and individual roads, where $W_{\mathrm{r}}$ is the extrusion width, $H_{\mathrm{r}}$ is layer height and $d_{\mathrm{r}}$ is raster-to-raster distance.

head's liquefier can be considered as a two-dimensional, axisymmetric, steady-state, advection-conduction heat transfer process [19]. Gaps between the filament and the wall of the liquefier, caused by the filament's diameter inconsistency can be expected to hinder heat transfer and result in irregular viscous behavior of the melt on the upper part of the liquefier. Diameter inconsistencies have adverse effects on the material 
Table 2. Fused filament fabrication toolpath and process parameters.

\begin{tabular}{|c|c|c|c|c|c|}
\hline \multicolumn{4}{|c|}{ Toolpath parameters } & \multicolumn{2}{|c|}{ Process parameters } \\
\hline Layer height (mm) & 0.1 & Speed for non-print moves $(\mathrm{mm} / \mathrm{s})$ & 100 & Filament diameter $(\mathrm{mm})$ & $1.78 \pm 0.04$ \\
\hline Vertical shells (\#) & 3 & Speed for print moves $(\mathrm{mm} / \mathrm{s})$ & 20 & Extrusion multiplier & 1 \\
\hline Horizontal shells (\#) & 0 & Infill/perimeters overlap (mm) & 0.15 & Extrusion temperature $\left({ }^{\circ} \mathrm{C}\right)$ & 205 \\
\hline Infill density (\%) & 100 & Extrusion width $(\mathrm{mm})$ & Equal to nozzle diameter & Fan speed $(\%)$ & 20 \\
\hline Infill pattern & Rectilinear & Infill/perimeters overlap (mm) & 0.1 & Disable fan for the first & 2 Layers \\
\hline Infill angle (degree) & 90 & Minimum detail resolution (mm) & 0.02 & & \\
\hline
\end{tabular}

feeding mechanism as well, since mismatches between the roller and filament surfaces may lead to filament slipping. Furthermore, the filament's mean diameter is used by the Computer Aided Manufacturing (CAM) program, which automatically calculates the material's feed velocity in the $3 \mathrm{D}$ printer's extruder. As a result, the diameter's standard deviation is related to volumetric flow rate fluctuations during the $3 \mathrm{D}$ printing process, which can alter the distance between adjacent fibers, causing insufficient bonding or fiber overlapping and thus reducing the physical object's dimensional accuracy and structural integrity.

\subsection{Computer aided manufacturing - toolpath and process parameters}

Computer aided manufacturing software typically enables control of several design and process parameters related to fused filament fabrication. Design parameters define the toolpath followed by the nozzle's tip and include individual fibers' width and height (commonly referred to as "road/extrusion width" and "layer's height/thickness", respectively), as well as various deposition strategies to form and fill the successive cross sections of the part. A common deposition strategy is to separately deposit one or more continuous contours of all boundary 2D surfaces included in a given cross section and to fill the space between them, (corresponding to the part's interior), with specific infill patterns. Part orientation in relation to infill orientation and to the system's main axes of movement ( $X Y Z$ for cartesian 3D printers) plays an important role in surface finish, dimensional accuracy, cost and mechanical behavior $[20,21]$. In the simple case of rectilinear infill pattern (Figure 3), each layer is filled with a raster of parallel roads and adjacent layers have a fixed $90^{\circ}$ raster angle between them. By adjusting infill density, which is expressed as a percentage of occupied space, more sparse or dense parts can be produced with bigger or smaller distances among contiguous fibers of the same layer (raster to raster distance, $d_{\mathrm{r}}$ ). A slightly negative $d_{\mathrm{r}}$, corresponding to fiber overlapping, has been reported to reduce void density and increase contact area among fibers and thus resulting in stronger fiber-to-fiber bonds [22, 23]. However, the excessive material buildup at the layer's perimeter significantly affects dimensional accuracy on the $X Y$ plane. As individual fibers are deposited on the previously solidified layer of the material, heat exchanges by conduction develop on contact surfaces between adjacent fibers and by convection and radiation with the surroundings. Upon the deposition of new layers, new physical contacts are generated; hence, several heat transfer modes change and heat transfer with air entrapped between contiguous filaments may also develop. Toolpath and G-code generation have a significant effect on thermal stress accumulation in fibers and layers and thus different CAM programs with the same input values, produce parts with different responses to external stresses. In this study, an open-source G-code generating program (Slic3r 1.2) was used for specimen fabrication. A second G-code generating program (Voxelizer 1.4), with a different generating algorithm, was used with the same input values of toolpath and process parameters, in order to estimate the effect of different CAM programs on the mechanical properties of the final part.

As far as process parameters are concerned, they include extrusion temperature, chamber temperature, cooling rate, filament feed velocity and volumetric flow rate, among others. The extrusion process does not have a considerable influence on the strength and modulus of the material, but notably affects the maximum strain, since during extrusion through the nozzle, polymer chains are submitted to stressinduced orientation, which reduces the elongation characteristics of the material [12]. The rate at which the filament is fed to the liquefier (feed velocity) is dynamically controlled and connected to velocity changes of the print head, in order to maintain a constant volumetric flow rate. The amount of melt which is present in the reservoir, the temperature of the melt, and consequently, the viscosity and surface energy of the melt, vary with feed rate [14]. In the case of constant linear movement of the print head, the extruder motor speed is proportionate to printing speed, so an indirect control of feed speed can be achieved varying printing speed for the same toolpath characteristics. Extrusion temperature and deposition rate are identified as the major parameters influencing inter- and intra-layer bonding [24].

User-defined processing parameters for G-code generation and specimen fabrication are listed in Table 2, which resulted from temperature and printing speed optimization, as well as adjustments on the standard processing profile for PLA recommended by the manufacturer. A constant layer thickness of $0.1 \mathrm{~mm}$ was used for all specimens and extrusion widths were determined by nozzle diameter. Three nozzles with the same design characteristics and $0.2,0.3,0.4 \mathrm{~mm}$ openings were tested and extrusion width was set equal to the respective nozzle diameter. Nozzle diameter, along with the viscosity of the melt, determine the pressure drop during extrusion and thus the force required from the feed mechanism. Pressure drop increases as nozzle diameter increases, or as feed velocity 

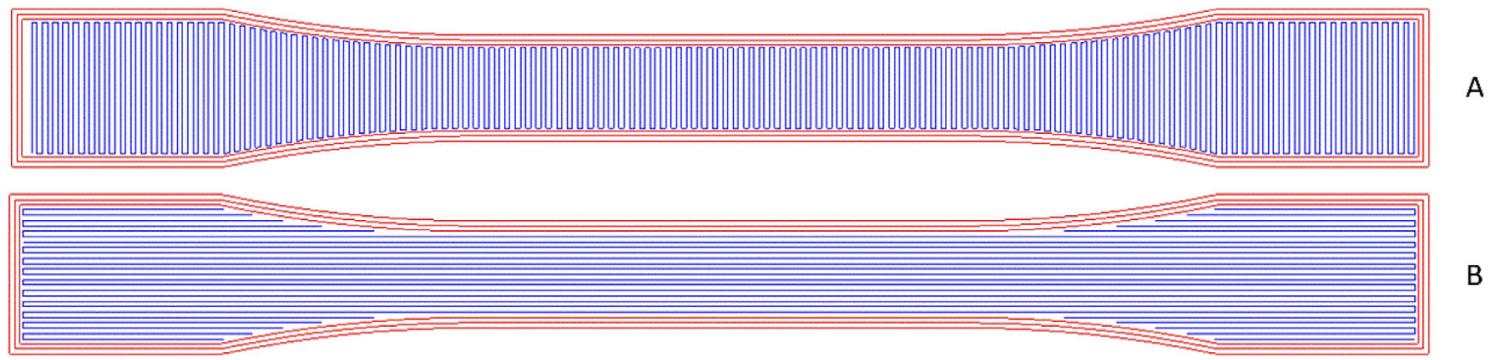

B

Figure 4. Toolpath simulation for tensile specimen fabrication with three contours and rectilinear infill pattern with $100 \%$ nominal density. Specimens consist of alternating Type A and Type B layers.
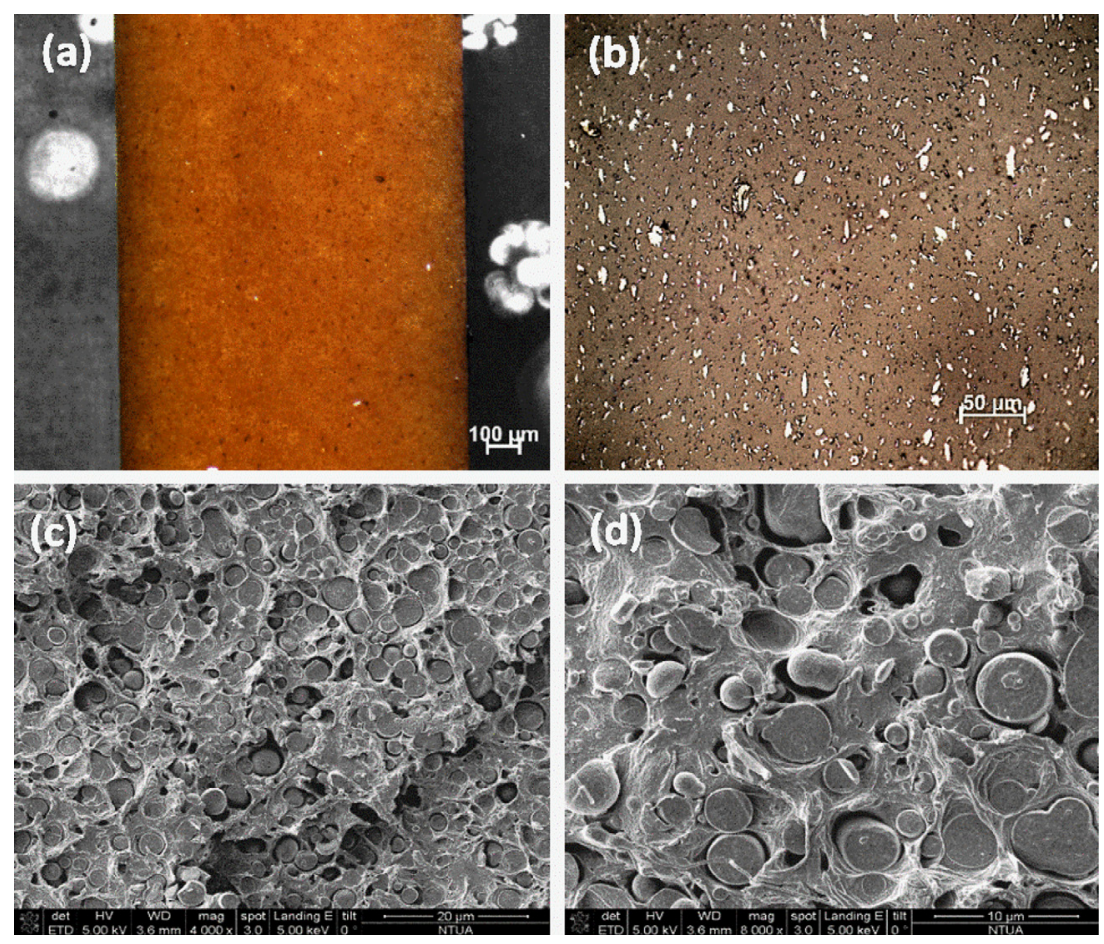

Figure 5. (a-b) Reflected light (brightfield and polarized illumination) micrographs of PLA/Lignin with $5 \%$ lignin (magnification $\times 100$ and $\times 500)$. (c-d) Closer examination $(\times 4000$ and $\times 8000$ magnification) of the morphology of lignin aggregation with SEM, from the tensilefractured surface of sample with $15 \%$ lignin.

increases. Furthermore, in the vicinity of the nozzle, the polymer melt is under stress and part of the deformation energy stored elastically leads to radial expansion of the fiber after extrusion. Individual fibers were extruded from each nozzle at three print speeds $(20,40$ and $60 \mathrm{~mm} / \mathrm{s})$. Surface morphology and diameter were studied at the center of the fibers, in order to avoid errors related to print head acceleration and deceleration. Since no standard test specimens exists for characterization of parts processed with FFF, tensile specimens were fabricated based on a 3D model of ASTM D638 Standard Test Method for Tensile Properties of Plastics, Type I specimen, designed with Autodesk Fusion $360 \mathrm{CAD}$ and extracted in fine quality Stereolithography (STL) format. The original specimen was scaled down by $\times 0.6$ to avoid fabrication errors near the boarders of the available working volume. To ensure the repeatability of the fabrication process, all tensile specimens were fabricated separately, with their wide surface parallel to the $X Y$ plane and gauge section parallel to $X$ axis, at the same position near the center of the build platform. Toolpath simulation generated by G-code generating software is depicted in Figure 4. Before the fabrication of each specimen, a touch probe sensor (by Zmorph LLC) was used for precise leveling of the build platform and the nozzle's distance from surface $(0.1 \mathrm{~mm})$ was verified at four points within the surface of specimen fabrication.

\section{Results}

\subsection{Bulk material characterization}

\subsubsection{Reflected light microscopy}

The morphological analysis of binary PLA/Lignin blends was performed with reflected light microscopy and scanning electron microscopy. In general, the morphology is defined by the complex thermomechanical history experienced by the 


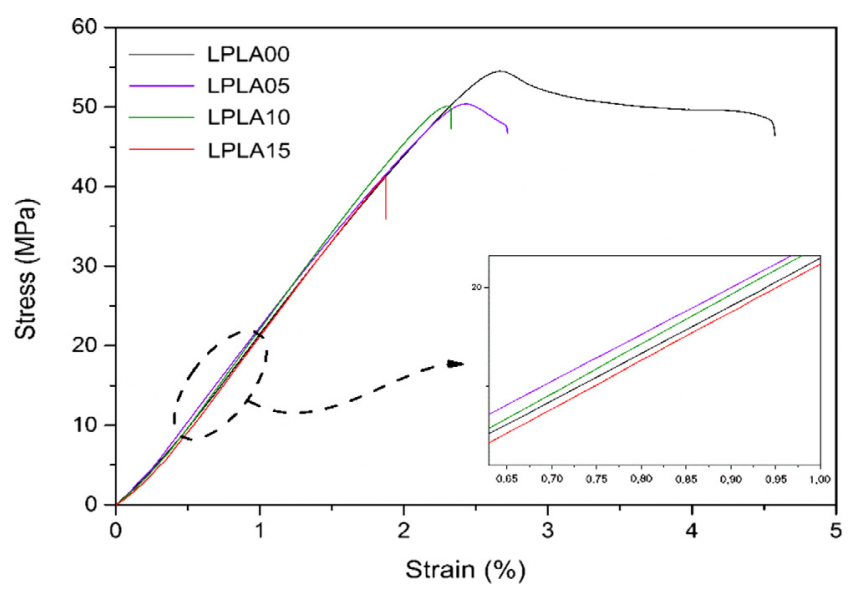

Figure 6. Engineering stress-strain curves of the PLA/Lignin blends with $0,5,10$ and 15 wt.\% lignin content.

Table 3. Tensile properties of PLA/Lignin composites.

\begin{tabular}{cccc}
\hline Sample & $E(\mathrm{GPa})$ & UTS $(\mathrm{MPa})$ & el $(\%)$ \\
\hline LPLA00 & $2.31 \pm 0.04$ & $55.9 \pm 0.6$ & $4.57 \pm 0.22$ \\
LPLA05 & $2.33 \pm 0.05$ & $50.3 \pm 0.9$ & $2.81 \pm 0.10$ \\
LPLA10 & $2.41 \pm 0.06$ & $50.1 \pm 0.5$ & $2.32 \pm 0.17$ \\
LPLA15 & $2.39 \pm 0.06$ & $41.3 \pm 0.5$ & $1.88 \pm 0.34$ \\
\hline
\end{tabular}

different constituents during processing [25]. All samples formed heterogeneous systems, due to the low compatibility between PLA matrix and the unmodified kraft lignin, which has been previously reported [24-28]. At 5 wt.\% lignin content, the morphology mainly consists of a uniform dispersion of lignin aggregates of small size $(<20 \mu \mathrm{m})$ in homogeneous surrounding matrix of PLA/Lignin, as it can be seen at Figures 5a and 5b. At higher lignin content, there is an increase in aggregates' concentration, which remained uniformly dispersed. At $20 \%$ lignin, particle size increases because of coalescence phenomena.

A closer examination of lignin aggregation at smaller scales can be achieved from SEM analysis of the fractured surface of the samples (Figures 5c and 5d). Prior to examination, sample surfaces were covered with gold sputter coating. It can be seen that lignin aggregates consist of smaller lignin domains of fairly circular shape, closely packed in specific regions of the PLA matrix. Interfacial separation and sliding between lignin's aggregates and the surrounding matrix are indicative of very weak secondary interactions between the two polymers.

\subsubsection{Tensile testing}

The type of morphology and the phase dimensions determine the mechanical and physical properties of the blend at large, which is influenced by intermolecular lignin-lignin, PLA-PLA and PLA-lignin interactions. Consequently, the observed immiscibility between the two constituents results in minor effective stress transfer between lignin's aggregates and PLA matrix and increases PLA's brittleness, which is shown as a significant reduction in the plastic region of stress-strain curves and disappearance of yield point. However,

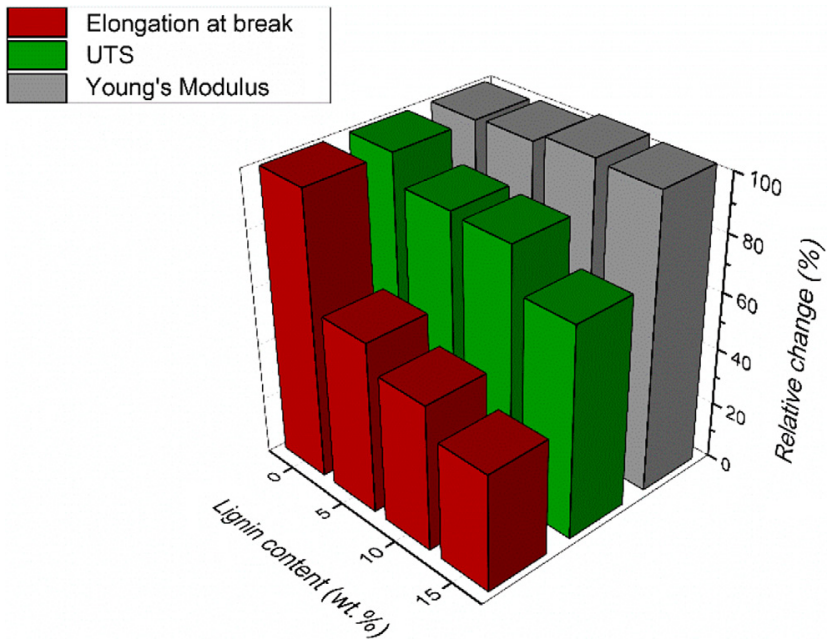

Figure 7. Percentage change of PLA's mechanical properties (ultimate tensile strength, Young's modulus of elasticity and elongation at break) with increasing lignin content.

lignin has no adverse effect on Young's modulus of elasticity, as it can be seen in Figure 6 and Table 3. Samples with 5 and $10 \mathrm{wt} . \%$ lignin displayed a $40 \%$ and $49 \%$ reduction in elongation at break respectively. The relative change in PLA's Young's modulus of Elasticity $(E)$, Ultimate Tensile Strength (UTS) and Elongation at Break (el) with increasing lignin content is presented in Figure 7. For samples LPLA10 and LPLA15, UTS is equal to breaking strength (absence of yield point), while the breaking strength of pure PLA and PLA with 5 wt.\% lignin is slightly smaller than UTS, at 48.1 and 47.6 MPa, respectively. The sample with $20 \mathrm{wt} . \%$ lignin content was too brittle for tensile specimens to be cut.

\subsubsection{Thermal characterization}

The DSC thermograms recorded during cooling and second heating of PLA composites are reported in Figure 8. A single glass transition temperature $\left(T_{\mathrm{g}}\right)$ registered for all blends, but no deduction can be made for the miscibility of the ingredients, since lignin's content is low and its $T_{\mathrm{g}}$ is possibly overlapped by PLA's melting peaks. At the chosen cooling rate $\left(10^{\circ} \mathrm{C} / \mathrm{min}\right)$, the material did not develop a significant crystalline component and no crystallization peaks were recorded during cooling cycle both for pure PLA and PLA/Lignin composites. Therefore, a highly amorphous structure is obtained with thermodynamic instability, which can form local ordered structure during annealing below glass transition temperature due to the enthalpic relaxation of the polymeric chains [29, 30], the melting of which is manifested as a small endothermic peak during the heating cycle after the glass transition process (Figure 8). When annealed between glass transition temperature $\left(T_{\mathrm{g}}\right)$ and melting temperature $\left(T_{\mathrm{m}}\right)$, the mobility of chain segments of glassy polymers increases gradually and cold crystallization occurs [31]. Lignin appears to promote PLA's double melting behavior, which is attributed to the melting of two populations of lamellae. The lower temperature peak is connected to the melting of small lamellae produced by secondary crystallization, while the higher temperature 
(a)

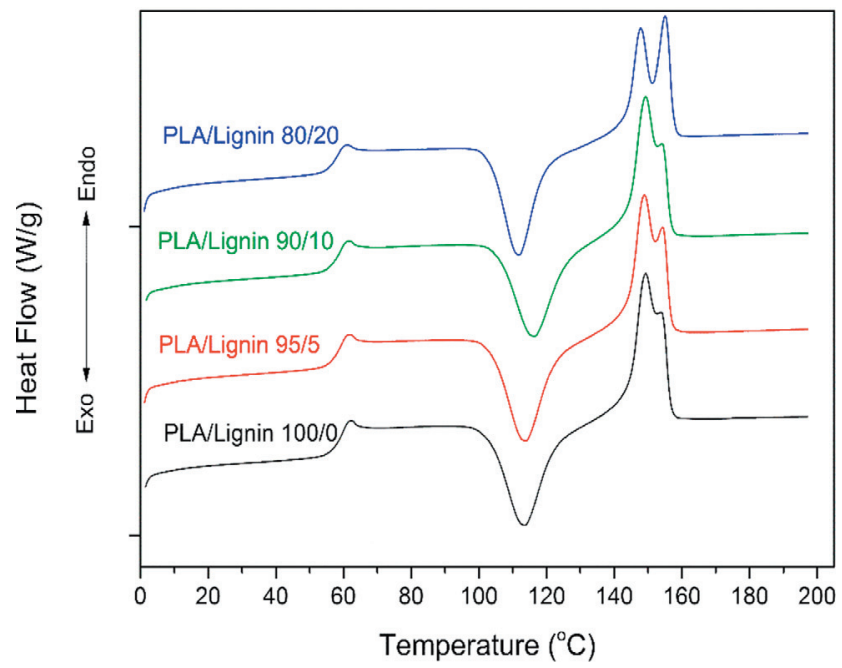

(b)

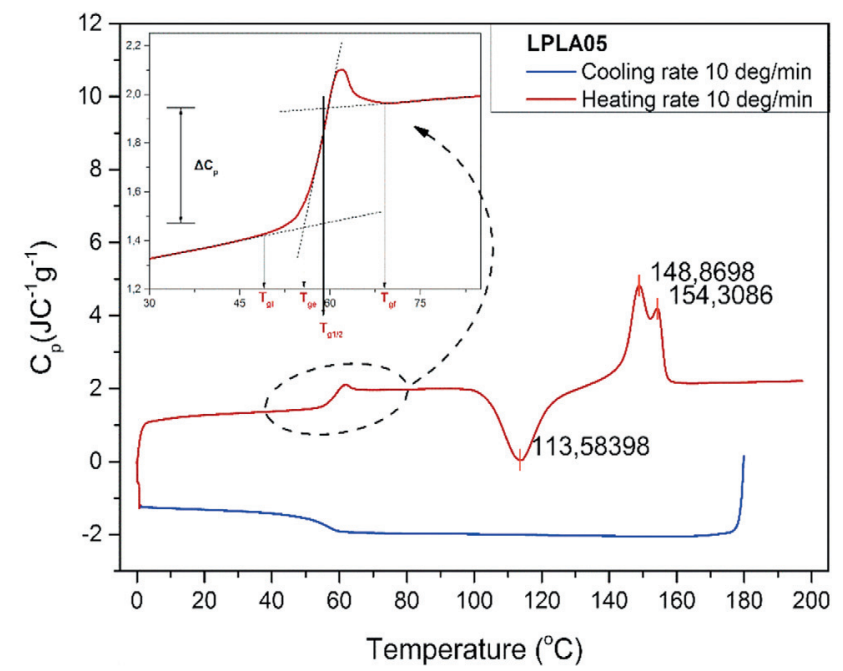

Figure 8. (a) DSC thermographs of PLA/lignin bulk composites. (b) DSC thermograph of sample with 5 wt.\% lignin. Quantities for characterization of the glass transition of sample containing $5 \mathrm{wt} . \%$ lignin: extrapolated onset temperature $\left(T_{\mathrm{ge}}\right)$, half-step temperature $\left(T_{\mathrm{g} 1 / 2}\right)$, change of the normalized heat capacity during transition $\left(\Delta C_{\mathrm{p}}\right)$, initial $\left(T_{\mathrm{gi}}\right)$ and final $\left(T_{\mathrm{gf}}\right)$ temperatures of the glass transition.

Table 4. Transition temperatures and associated changes in heat capacity, enthalpy and crystallinity of PLA/Lignin composites.

\begin{tabular}{ccccccccccc}
\hline Sample & $T_{\mathrm{gi}}\left({ }^{\circ} \mathrm{C}\right)$ & $T_{\mathrm{gf}}\left({ }^{\circ} \mathrm{C}\right)$ & $T_{\mathrm{g} 1 / 2}\left({ }^{\circ} \mathrm{C}\right)$ & $T_{\mathrm{ge}}\left({ }^{\circ} \mathrm{C}\right)$ & $\Delta C_{\mathrm{p}}\left(\mathrm{JC}-1 \mathrm{~g}^{-1}\right)$ & $T_{\mathrm{cc}}\left({ }^{\circ} \mathrm{C}\right)$ & $T_{\mathrm{m} 1}\left({ }^{\circ} \mathrm{C}\right)$ & $T_{\mathrm{m} 2}\left({ }^{\circ} \mathrm{C}\right)$ & $\Delta H_{\mathrm{cc}}(\mathrm{J} / \mathrm{g})$ & $\Delta H_{\mathrm{m}}(\mathrm{J} / \mathrm{g})$ \\
\hline LPLA00 & 47.4 & 71.0 & 59.2 & 56.0 & 0.52 & 113.4 & 149.2 & 153.9 & -25.0 \\
LPLA05 & 49.0 & 69.0 & 57.4 & 55.7 & 0.48 & 113.6 & 148.9 & 154.3 & -24.7 & 25.9 \\
LPLA10 & 48.0 & 70.0 & 59.0 & 55.0 & 0.46 & 116.3 & 149.3 & 154.2 & -24.4 & 25.0 \\
LPLA20 & 45.5 & 69.0 & 57.3 & 54.7 & 0.43 & 111.8 & 148.2 & 155.0 & -22.3 & 22.4 \\
\hline
\end{tabular}

peak originates from the melting of major crystals formed in the primary crystallization process. As part of the ongoing research related to crystalline behavior of semi-crystalline polymers, it has been suggested that the primary crystals are first formed, followed by the formation of secondary crystals and simultaneous thickening of the primary crystals [32]. Other authors have explained double melting behavior with a melt-recrystallization model, where the low-temperature and high-temperature peaks in the DSC curve are attributed to the melting of some amount of the original crystals and to the melting of crystals formed through a melt-recrystallization process during the heating scan, respectively [33]. As lignin content increases, there is a greater contribution from the melting of major crystals to the specific melting enthalpy of the sample or from crystals formed through a melt-recrystallization process, which is implied by the increase in the higher temperature melting peak in Figure 8. Therefore, for the given heating rate lignin affects nucleation and crystal growth, possibly encouraging recrystallization or the formation of thicker crystalline structures during the cold crystallization process.

Basic quantities which characterize the samples' glass transition, cold crystallization and melting process were derived from DSC thermographs and are listed in Table 4. No significant changes were observed for the initial $\left(T_{\mathrm{gi}}\right)$, final $\left(T_{\mathrm{gf}}\right)$, extrapolated onset $\left(T_{\mathrm{ge}}\right)$ and half-step $\left(T_{\mathrm{g} 1 / 2}\right)$ glass transition temperature and for the associated change in heat capacity $\left(\Delta C_{\mathrm{p}}\right)$ before and after glass transition. The peak temperature

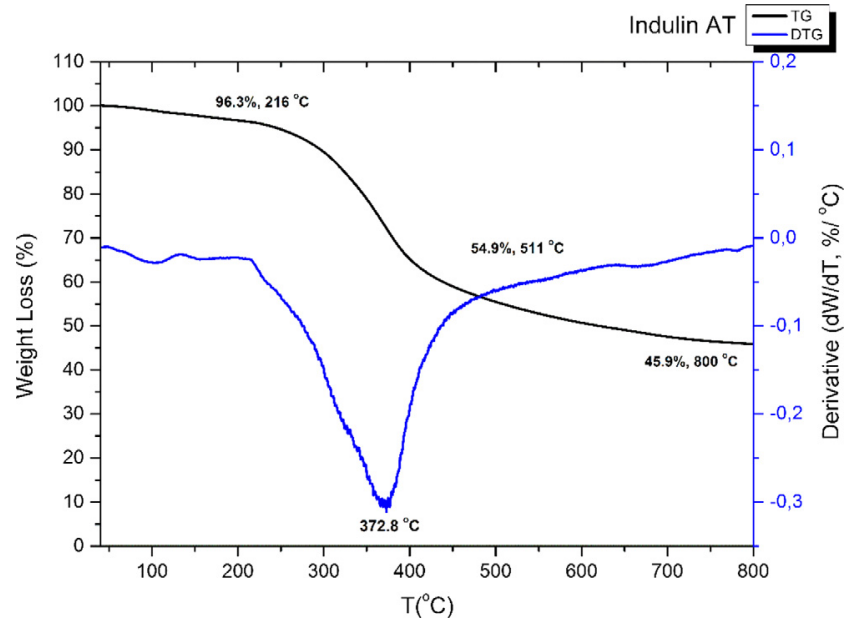

Figure 9. TGA plot of Indulin AT kraft lignin and the derivative TGA curve, obtained under nitrogen atmosphere at $10^{\circ} \mathrm{C} / \mathrm{min}$ heating rate.

for cold crystallization $\left(T_{\mathrm{cc}}\right)$, low melting peak $\left(T_{\mathrm{m} 1}\right)$, high melting peak $\left(T_{\mathrm{m} 2}\right)$ and the related cold crystallization and melting enthalpies also seem to be independent from lignin content, with very small variations. As a result, no special intermolecular interaction between PLA and lignin can be confirmed, in accordance to the observed phase morphology. 

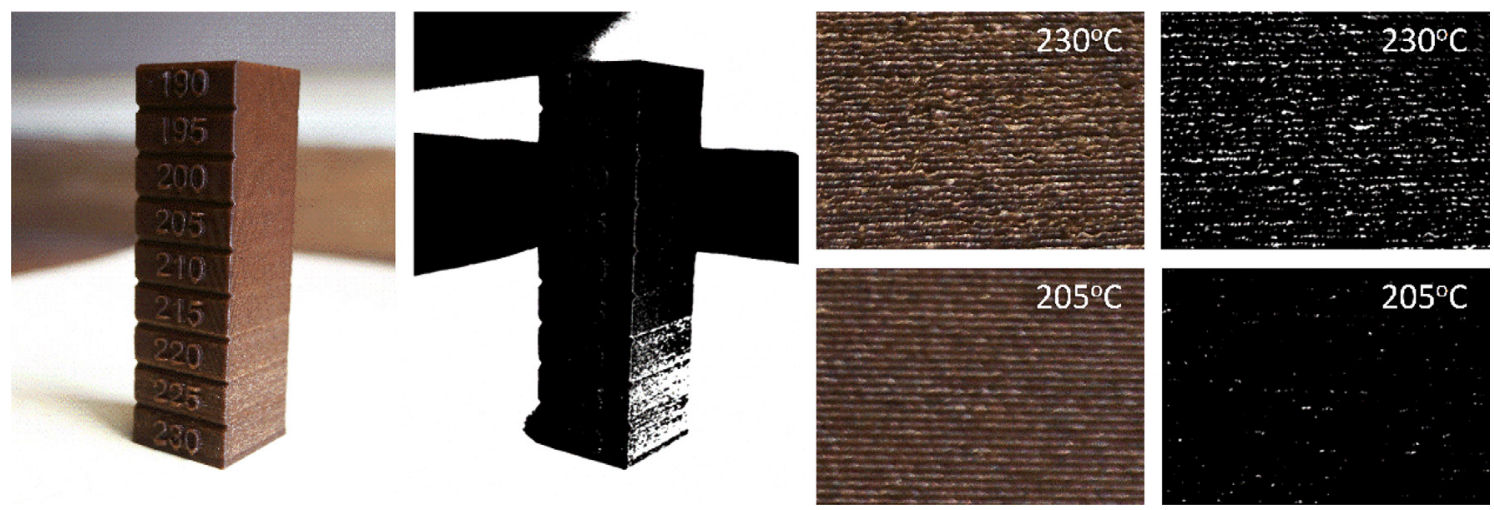

Figure 10. Macrographs of temperature calibration specimen captured with Sony SLT-A57 SAL-1855, depicting changes on surface roughness caused by thermal degradation (in black/white contrasted images, from darker to brighter images.

Table 5. Characteristic decomposition temperatures of Indulin AT kraft lignin.

\begin{tabular}{cccccccc}
\hline Sample & $T_{\mathrm{o}}$ & $T_{\text {end }}$ & $T_{1}$ & $T_{2}$ & $T_{3, \max }$ & $T_{5 \%}$ & $T_{30 \%}$ \\
\hline Kraft lignin & 216 & 511 & 101.6 & 156.5 & 372.8 & 237 & 379 \\
\hline
\end{tabular}

Furthermore, since the FFF process involves melt quenching at higher cooling rates and instant solidification of the material after its deposition, it can be deducted that the material's crystallinity during and after FFF processing will mainly be defined by stress-induced crystallization.

The Thermogravimetry (TG) (in weight loss percentage) and Derivative Thermogravimetry (DTG) (in weight loss percentage per ${ }^{\circ} \mathrm{C}$ ) curves of Indulin AT kraft lignin, obtained at $10{ }^{\circ} \mathrm{C} / \mathrm{min}$ heating rate under nitrogen atmosphere, are plotted in Figure 9. Thermal degradation data indicates weight loss and the first derivative (DTG) indicates the corresponding rate of weight loss. The peak of DTG can be presented as a measure of thermal decomposition and can be used as a means to compare thermal stability characteristics of different materials. As it can be seen in Figure 10, thermal decomposition occurred over a wide temperature range, starting at approximately $216{ }^{\circ} \mathrm{C}\left(T_{\mathrm{o}}\right)$. Lignin's weight loss percentages for various characteristic decomposition temperatures are summarized in Table 5, where $T_{\mathrm{o}}$ is initial decomposition temperature, $T_{\text {end }}$ is terminal decomposition temperature, $T_{1}$ and $T_{2}$ are attributed to the evolution of humidity and chemically bound water respectively, $T_{3, \max }$ is maximum decomposition temperature corresponding to DTG peak and $T_{5 \%}, T_{30 \%}, T_{50 \%}$ are temperatures of $5,10,30$ and $50 \%$ weight loss due to degradation.

\subsection{Fused filament fabrication process optimization}

\subsubsection{Extrusion temperature}

A temperature calibration specimen, consisting of a $20 \times 20 \times 80 \mathrm{~mm}$ hollow rectangular cuboid with 0.8 cell thickness, was modeled in Autodesk Fusion 360 CAD program and extracted in STL file format, in order to assess the impact of liquefier temperature on surface finish (Figure 10). Extrusion temperature varied from 230 to $190{ }^{\circ} \mathrm{C}$, with $5{ }^{\circ} \mathrm{C}$ steps every $1 \mathrm{~cm}$ across $Z$ axis (10 layers with $0.1 \mathrm{~mm}$ height). A brass nozzle with $0.4 \mathrm{~mm}$ diameter was used for specimen fabrication and the rest of the user-defined input values for the CAM program are listed in Table 2. It has been observed that the $3 \mathrm{D}$ printing extrusion temperature is generally higher than that needed for filament extrusion [34], due to the material's short residence time in the liquefier's reservoir and the limited power of the feeding system's stepper motor (NEMA 17), compared to the single-screw extruder. Also, the liquefier temperature set on CAM program reflects the temperature of the melt reservoir on the upper part of the nozzle, close to the print head's temperature sensor. The correct deposition temperature is expected to be lower, since the tip of the brass nozzle itself is not heated and insulated [35]. From the images of Figure 10, it can be seen that surface roughness is directly connected to the material's thermal stability. At temperatures higher than $215^{\circ} \mathrm{C}$, the anisotropy of surface's topography increases, in accordance to lignin's thermal decomposition pattern (Figure 9). At $190{ }^{\circ} \mathrm{C}$, the melt's viscosity is significantly higher, and lignin's agglomerates hinder material flow, resulting in nozzle clogging. The optimum combination of surface roughness and material flow was achieved at $205^{\circ} \mathrm{C}$.

\subsubsection{Extrusion of individual fibers and infill inspection}

Both PLA and PLA with 5 wt.\% lignin fibers with $15 \mathrm{~cm}$ length were individually extruded from the FFF system at $205^{\circ} \mathrm{C}$, using nozzles with different openings $(0.4,0.3$, $0.2 \mathrm{~mm}$ ). From Figures 11 and 12, it is evident that lignin causes a severe increase in fibers' surface roughness (in comparison with pure PLA fibers) and the actual extrusion width is mainly defined by the size and distribution of lignin aggregates. Phase separation is more intense in filament as opposed to the bulk material with the same lignin content, due to the dispersive mixing limitations of the single-screw system used for filament production, as to the twin-screw internal 

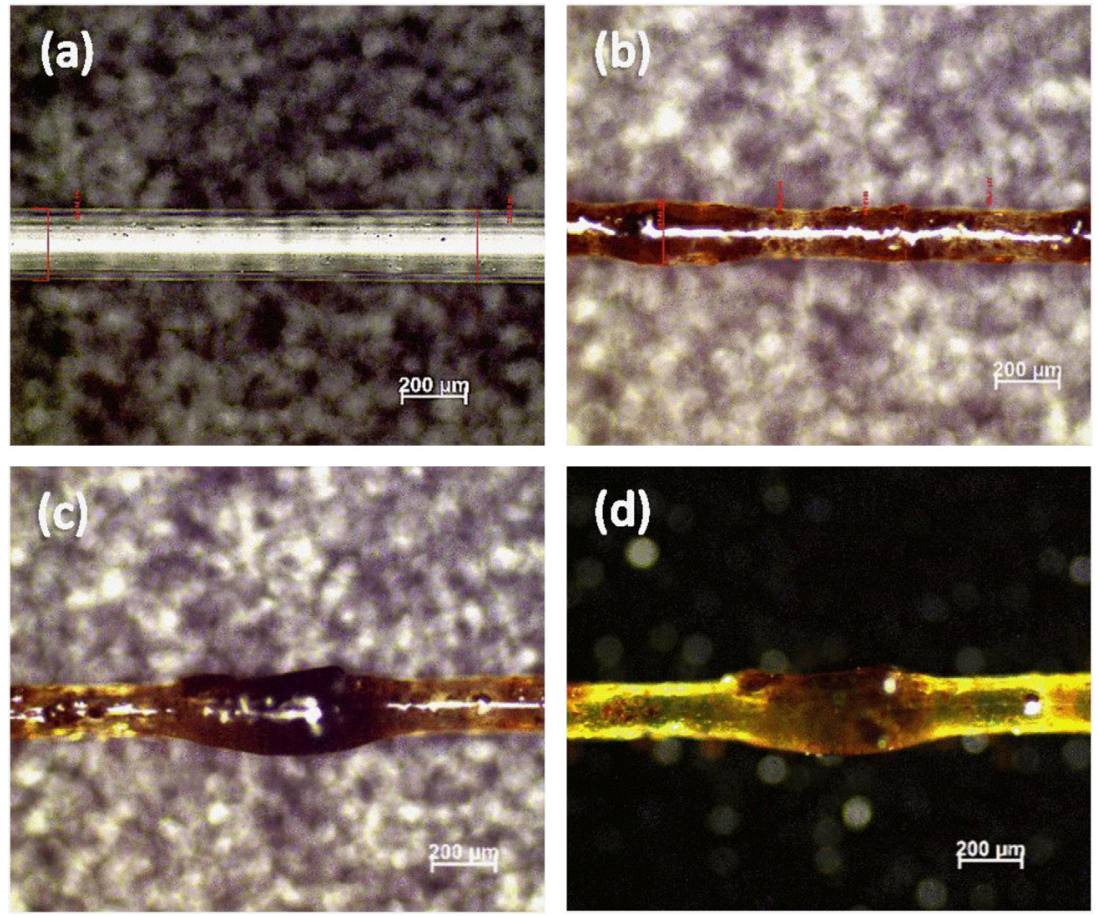

Figure 11. Individual fibers extruded from $0.2 \mathrm{~mm}$ nozzle (a-b) $20 \mathrm{~mm} / \mathrm{s}$ printing speed and (c-d) $60 \mathrm{~mm} / \mathrm{s}$, where lignin aggregation causes up to $30 \%$ increase in fiber's diameter, respectively.
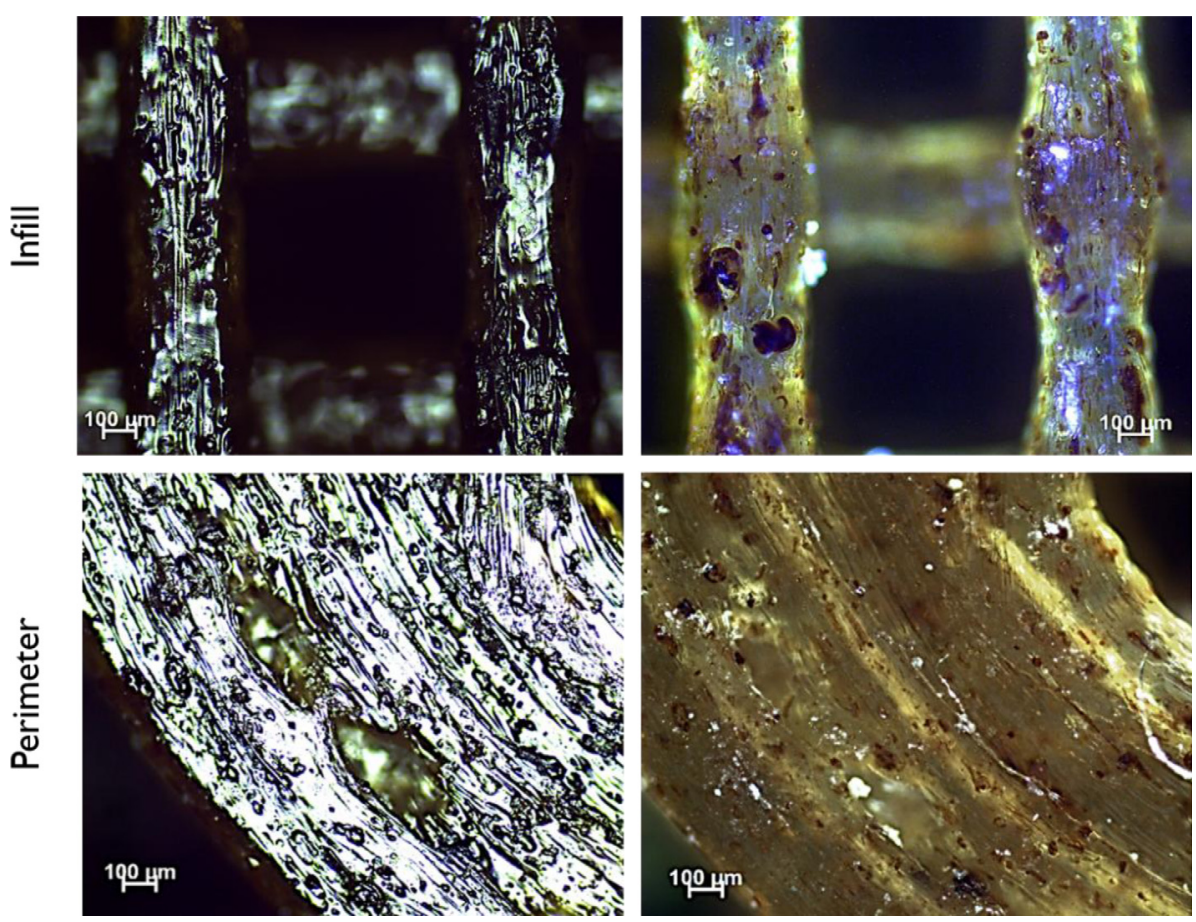

Figure 12. Reflected light (brightfield and polarized illumination) micrographs of specimen with $50 \%$ rectilinear infill density and three successive contours, for inspection of lignin dispersion, surface texture and volumetric flow fluctuations.

compounder used for sample preparation. For the tested print velocities $(20,40$ and $60 \mathrm{~mm} / \mathrm{s})$, no nozzle clogging occurred. However, as nozzle diameter decreases and becomes comparable with the size of lignin's aggregates, diameter inconsistencies become more prominent at higher speeds, as it can be seen in Figures 11c and 11d, where the local diameter increases by $30 \%$. Therefore, $20 \mathrm{~mm} / \mathrm{s}$ speed was selected as a common printing speed for all nozzles. A cylindrical specimen 
(a)

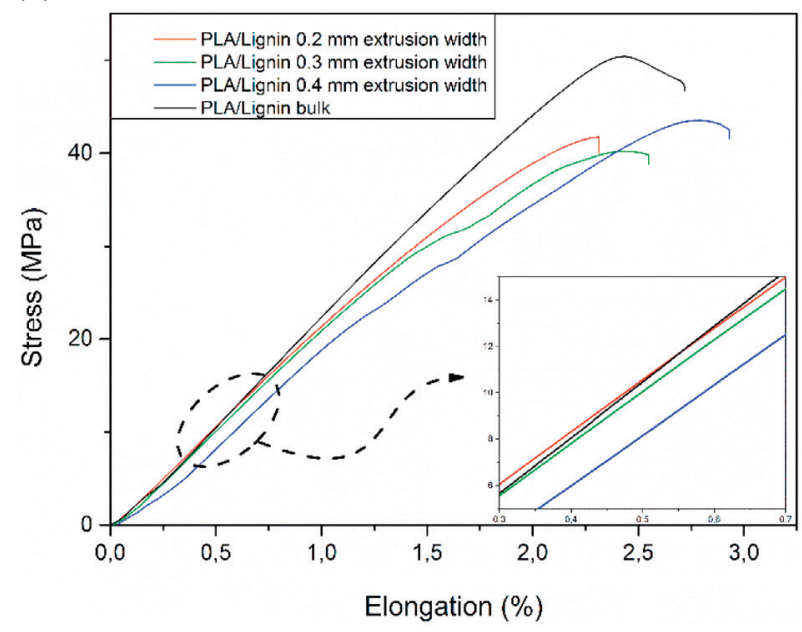

(b)

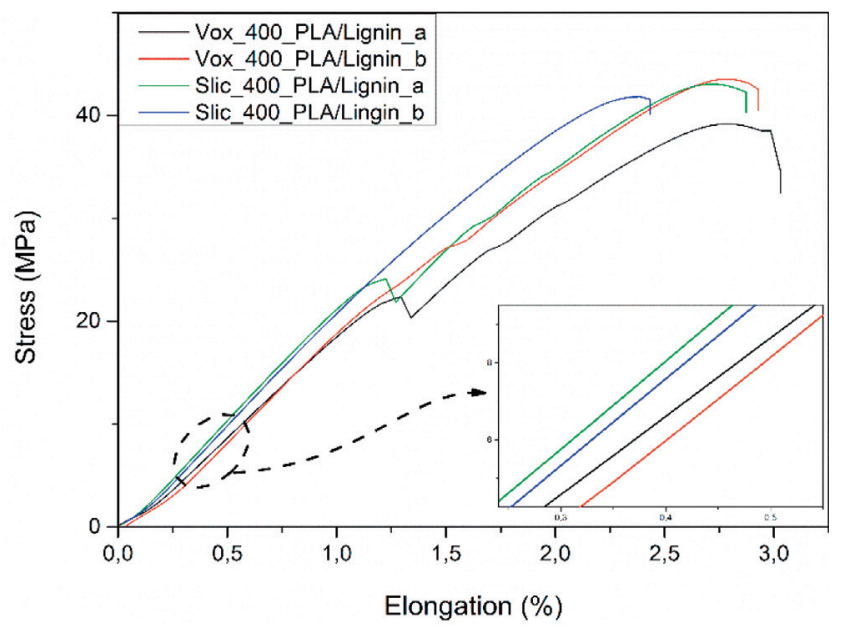

(c)

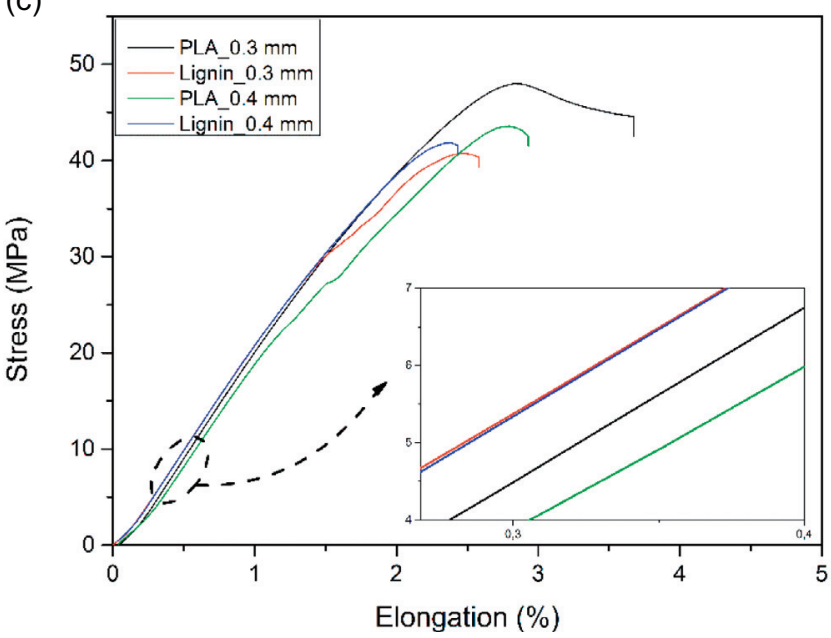

Figure 13. (a) Effect of different nozzle diameter on specimen's tensile properties. Comparison with the tensile properties of bulk material with the same (5 wt.\%) lignin content. (b) Effect of different CAM programs on tensile properties of specimens with the same toolpath and process parameters. Representative examples with and without premature internal failure. (c) Tensile properties of specimens produced from pure PLA and PLA/Lignin filament for two different nozzle diameters.

with $1 \mathrm{~cm}$ radius and $1 \mathrm{~cm}$ height, with 3 contours and rectilinear infill pattern (50\% nominal density) was fabricated with $0.4 \mathrm{~mm}$ extrusion width for infill inspection. Uneven distribution of lignin phases of various sizes is depicted at Figure 12, along with a case of over-extrusion dew to volumetric flow fluctuations during the extrusion of the contour of the specimen.

\subsubsection{Tensile properties of $3 \mathrm{D}$ printed specimens}

As it has been noted by other studies [12, 13], small variations of the processing conditions can strongly affect specimens mechanical properties. The reported values and standard deviations are the average of five specimens, which failed within the specimen's gauge section. The effect of different extrusion widths is presented in Figure 13a and Table 6. As it has been mentioned, extrusion through different nozzles negatively affects specimen's maximum strain (and increases its standard deviation from average value), because of the imponderable effect of the increased stress-induced orientation of polymer chains $[12,21]$. The $0.4 \mathrm{~mm}$ diameter nozzle resulted in specimens with maximum elongation at break similar to that exhibited by bulk material. Ultimate tensile strength and Young's modulus of elasticity have similar values for different road widths and are $18 \%$ and $6 \%$ reduced respectively, compared to bulk material. Figure 13b and Table 7 present the tensile properties of representative specimens fabricated with two different G-code generation programs and the same user-defined process and toolpath parameters. Two characteristic responses to tensile stresses appeared in both sets of tensile specimens, indicating that specimens fabricated under the same conditions can exhibit different fracture behaviors, because of premature inter- and intra-laminar failures related to over- or under-extrusion or weak bonds among individual fibers, especially at the fusion points between the infill and the internal contour. This can be also observed with SEM 
Table 6. Tensile properties PLA/Lignin 3D printed specimens with various extrusion widths - comparison with the bulk material.

\begin{tabular}{lccrr}
\hline \multicolumn{1}{c}{ Sample } & Extrusion width $(\mathrm{mm})$ & $E(\mathrm{GPa})$ & UTS $(\mathrm{MPa})$ & el $(\%)$ \\
\hline PLA/Lignin filament & 0.2 & $2.18 \pm 0.06$ & $41.8 \pm 1.6$ & $2.31 \pm 0.16$ \\
PLA/Lignin filament & 0.3 & $2.20 \pm 0.18$ & $40.2 \pm 0.7$ & $2.55 \pm 0.19$ \\
PLA/Lignin filament & 0.4 & $2.16 \pm 0.10$ & $43.6 \pm 2.1$ & $2.83 \pm 0.26$ \\
PLA/Lignin bulk material & - & $2.33 \pm 0.05$ & $50.3 \pm 0.9$ & $2.81 \pm 0.10$ \\
\hline
\end{tabular}

Table 7. Tensile properties of representative PLA with 5 wt.\% lignin specimens, with and without premature failure, exhibited for both CAM programs used for fabrication.

\begin{tabular}{|c|c|c|c|c|c|c|}
\hline Sample & CAM program & $E(\mathrm{GPa})$ & $S_{\mathrm{pf}}(\mathrm{MPa})$ & $\mathrm{El}_{\mathrm{pf}}(\%)$ & UTS (MPa) & el $(\%)$ \\
\hline Vox_PLA/Lignin a & Voxelizer & 2.03 & 22.4 & $1.30-1.34$ & 39.2 & 2.99 \\
\hline Vox_PLA/Lignin b & Voxelizer & 2.16 & - & - & 43.6 & 2.93 \\
\hline Sli_PLA/Lignin a & Slic3r & 2.25 & 24.0 & $1.23-1.28$ & 43.1 & 2.88 \\
\hline
\end{tabular}
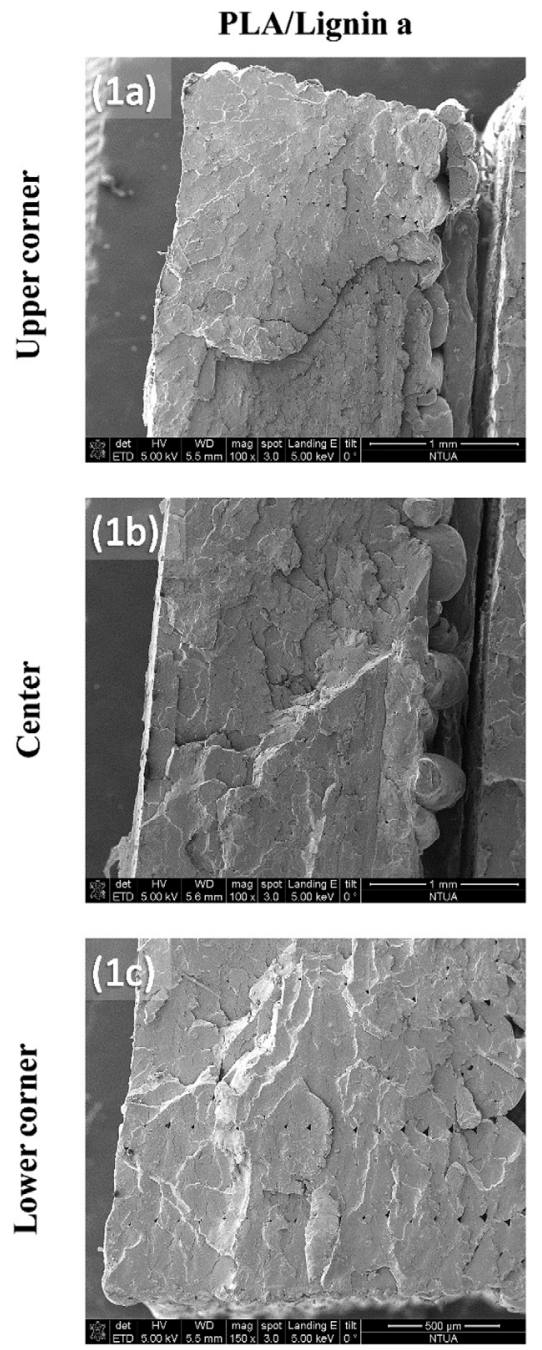

PLA/Lignin b
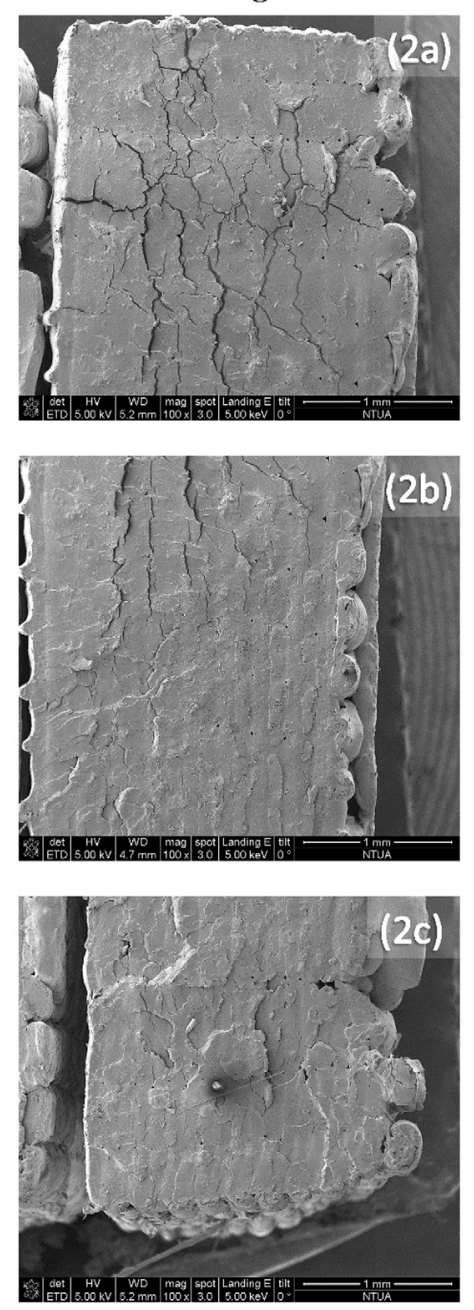

PLA
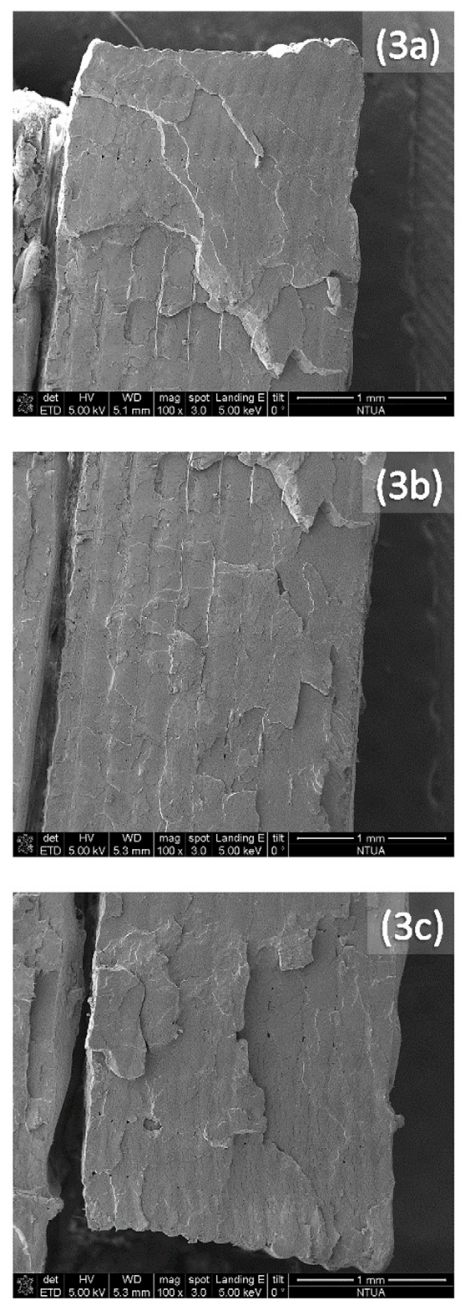

Figure 14. SEM micrographs of the fractured surface of 3D printed tensile specimens. The upper/lower corners of gauge region cross section consist of three successive contours. PLA/Lignin a and PLA/Lignin b, which were fabricated with different CAM programs, exhibit different fracture morphologies.

analysis of the fractured surfaces of tensile specimens fabricated with different CAM programs (Figure $14-1 \mathrm{a}-1 \mathrm{c}$ and $2 \mathrm{a}-2 \mathrm{c})$. The stress around which premature failure occurs is approximately half of the corresponding ultimate tensile strength and is noted as $S_{\mathrm{pf}}$ in Table $7 . \mathrm{El}_{\mathrm{pf}}$ is the strain region corresponding to negative slope of stress-strain curve. 
Table 8. Tensile properties of specimens fabricated from pure PLA and PLA/Lignin filament.

\begin{tabular}{|c|c|c|c|c|}
\hline Sample composition & Extrusion width (mm) & $E(\mathrm{GPa})$ & $\operatorname{UTS}(\mathrm{MPa})$ & el $(\%)$ \\
\hline PLA & 0.3 & $2.17 \pm 0.12$ & $48.2 \pm 0.9$ & $3.68 \pm 0.35$ \\
\hline PLA with 5 wt.\% Lignin & 0.3 & $2.21 \pm 0.33$ & $40.9 \pm 0.9$ & $2.58 \pm 0.16$ \\
\hline PLA & 0.4 & $2.13 \pm 0.08$ & $43.6 \pm 1.7$ & $2.93 \pm 0.21$ \\
\hline
\end{tabular}

Figure $13 \mathrm{c}$ and Table 8 present a comparison of tensile properties among identical specimens fabricated from pure PLA and PLA/lignin filaments. As the tensile stress increases, the failure will begin at the weakest raster and next weakest raster will break, in sequence, until total failure of the sample [36]. As expected, lignin addition mainly reduces elongation at break and increases PLA's brittle behavior, which can be also deducted by comparing the characteristics of PLA's fractured surface (Figure $14-3 \mathrm{a}-3 \mathrm{c}$ ) with PLA/Lignin specimens. PLA's interlayer fusion at the infill region appears to be improved every two layers, which is caused by toolpath variation (Figure 4), since the fabrication of Type-A layers has a reduced time between successive depositions of fibers and temperature at the bonding site remains higher for a longer period of time, improving neck formation and growth with the preceding layer $[37,38]$. Interestingly, this effect is limited in PLA/Lignin specimens, where all layers seem to have similar bond quality, with more complex fracture mechanisms. The non-selective trans-layer and trans-fiber crack propagation is indicative of improved bond quality, which could be explained by the adhesion-enhancing effect of lignin addition, caused by the increase in surface roughness of individual fibers [38]. As it has been mentioned, crack initiation and propagation is more probable to occur in specimen's upper/lower corners and more specifically in contour region, where void density is higher, or at the infill-contour fusion points. For the chosen process and toolpath parameters, very good adhesion among contiguous fibers and successive layers was achieved.

\section{Conclusions}

In this study, the properties of biobased PLA/Lignin blends were examined and correlated to processing conditions with fused filament fabrication and tensile properties of the produced parts. Samples with different PLA/lignin weight ratios were prepared and their phase morphology as well as mechanical and thermal properties were examined. It was found that all samples formed heterogeneous systems, where interfacial separation and sliding between lignin's aggregates and the surrounding PLA matrix are indicative of very weak secondary interactions between the two polymers. Phase separation also results in minor effective stress transfer between lignin's aggregates and PLA matrix, which increases PLA's brittleness and causes a significant reduction in the plastic deformation region of stress-strain curves and disappearance of yield point. However, lignin has no adverse effect on Young's modulus of elasticity. Furthermore, the material's slow crystallization rate (at $10^{\circ} \mathrm{C} / \mathrm{min}$ cooling rate no crystallization peaks were recorded), suggests that its crystallinity during and after FFF processing will mainly be defined by stress-induced crystallization. Lignin appears to promote PLA's double melting behavior and affects nucleation and crystal growth, possibly encouraging recrystallization or the formation of thicker crystalline structures during the cold crystallization process. Thermogravimetric analysis of kraft lignin indicates that its thermal decomposition occurred over a wide temperature range, starting at approximately $216^{\circ} \mathrm{C}$. Since increasing lignin content results in severe agglomeration and increase in the material's brittleness and melt's apparent viscosity during extrusion, the composition with $5 \mathrm{wt} . \%$ lignin content was selected for the production of 3D printing filament and segments of $1.78 \pm 0.04 \mathrm{~mm}$ diameter were used for extrusion of individual fibers and fabrication of tensile specimens. The addition of lignin causes a severe increase in fibers' surface roughness (in comparison with pure PLA fibers) and the actual extrusion width is mainly defined by the size and uneven distribution of lignin aggregates. Based on the material's response during processing, the optimum extrusion temperature $\left(205^{\circ} \mathrm{C}\right)$ and printing speed $(20 \mathrm{~mm} / \mathrm{s})$ for extrusion through nozzles with different diameters $(0.2,0.3$ and $0.4 \mathrm{~mm})$ were used for tensile specimen fabrication. Extrusion through different nozzles negatively affects specimen's maximum strain (and increases its standard deviation from average value), because of the imponderable effect of the increased stress-induced orientation of polymer chains. The $0.4 \mathrm{~mm}$ diameter nozzle resulted in specimens with maximum elongation at break similar to that exhibited by bulk material. Ultimate tensile strength and Young's modulus of elasticity have similar values for different road widths and are $18 \%$ and $6 \%$ reduced respectively, compared to bulk material. It was also found that specimens fabricated under the same conditions can exhibit different fracture behaviors, because of premature inter- and intra-laminar failures related to over- or under-extrusion or weak bonds among individual fibers, especially at the fusion points between the infill and the internal contour. The non-selective trans-layer and trans-fiber crack propagation observed in SEM micrographs of the fractured surface is indicative of improved bond quality, which could be explained by the adhesion-enhancing effect caused by the increase in surface roughness of individual PLA/Lignin fibers compared to pure PLA. Additionally, specimens fabricated with different CAM programs with the same input values for basic toolpath and processing parameters, exhibit different fracture morphologies, which implies that G-code generation algorithms also affect the final part's mechanical properties.

Future studies will focus on employing Design Of Experiment (DOE) techniques for systematic analysis and determination of the relation among basic toolpath and processing parameters and output measures, aiming for an overall material-based optimization of the FFF process. As far as the raw 
material is concerned, various methods for improving PLA compatibility with kraft lignin will be tested (addition of plasticizers and compatibilizing agents, use of lignin fractions with lower polydispersity indices, lignin chemical treatment) and their effect on the material's dynamic rheological properties will be examined. Also, thermal stability under oxidative and nonoxidative conditions, as well as thermal degradation during processing will be investigated.

Acknowledgements. This work has partially received funding from the European Union's Horizon 2020 research and innovation program MODCOMP under grant agreement No 685844 and EU FP7 Project "Functionalized Innovative Carbon Fibres Developed from Novel Precursors with Cost Efficiency and Tailored Properties" (FIBRALSPEC) under Grant Agreement No. 604248.

\section{References}

1. F.G. Calvo-Flores, et al., Applications of modified and unmodified lignins, in: Lignin and lignans as renewable raw materials, John Wiley \& Sons Ltd, New York City, United States, 2015, pp. 247-288.

2. H. Chung, N.R. Washburn, 2 - Extraction and types of lignin, in: Lignin in polymer composites, William Andrew Publishing, New York City, United States, 2016, pp. 13-25.

3. J.A.G. Ochoa de Alda, Resources, Conservation and Recycling 52 (2008) 965-972.

4. H. Naegele, et al., 13 - Applications of lignin materials and their composites (lignin applications in various industrial sectors, future trends of lignin and their composites), in: Lignin in polymer composites, William Andrew Publishing, New York City, United States, 2016, pp. 233-244.

5. Y.-L. Chung, et al., ACS Sustainable Chemistry \& Engineering 1 (2013) 1231-1238.

6. MarketsandMarkets, 3D printing plastic market by type (photopolymers, abs, pla, polyamide/nylon, others), by form (filament, ink, powder), by application, by end-user industry, and by region - global forecasts to 2020, 2016, Available from: http:// www.marketsandmarkets.com/Market-Reports/3d-printingplastic-market-21707470.html.

7. H. Tsuji, Poly (lactic acid), in: Bio-based plastics, John Wiley \& Sons Ltd, New York City, United States, 2013, pp. 171-239.

8. O. Faruk, et al., 6 - Lignin Reinforcement in Thermoplastic Composites, in Lignin in Polymer Composites, William Andrew Publishing, New York City, United States, 2016, pp. 95-118.

9. A. Bellini, S.U. Güçeri, M. Bertoldi, Journal of Manufacturing Science and Engineering 126 (2004) 237.

10. I. Gibson, W.D. Rosen, B. Stucker, Extrusion-Based Systems, in: Additive manufacturing technologies: rapid prototyping to direct digital manufacturing, Boston, MA, Springer US, 2010, pp. 160-186.

11. C. Bellehumeur, et al., Journal of Manufacturing Processes 6 (2004) 170-178.
12. A. Bellini, S. Güçeri, Rapid Prototyping Journal 9 (2003) 252-264.

13. M. Bertoldi, M.A. Yardimici, C.M. Pistor, S.I. Güçeri, G. Sala, Mechanical characterization of parts processed via fused deposition, in: Solid Freeform Fabrication Proceedings, Austin, TX, 1998.

14. B.N. Turner, R. Strong, S.A. Gold, Rapid Prototyping Journal 20 (2014) 192-204.

15. N. Sa'ude, M. Ibrahim, M.H.I. Ibrahim, Applied Mechanics and Materials 660 (2014) 89-93.

16. N. Mostafa, et al., Tsinghua Science and Technology 14 (2009) 29-37.

17. J.F.M. de Almeida, et al., Polymer Bulletin 73 (2016) 3531-3545.

18. N. Venkataraman, et al., Rapid Prototyping Journal 6 (2000) 244-253.

19. L.B. Ji, T.R. Zhou, Advanced Materials Research 97-101 (2010) 2585-2588.

20. M. Domingo-Espin, et al., Materials \& Design 83 (2015) 670-677.

21. B.N. Turner, S.A. Gold, Rapid Prototyping Journal 21 (2015) 250-261.

22. A.K. Sood, R.K. Ohdar, S.S. Mahapatra, Journal of Advanced Research 3 (2012) 81-90.

23. M. Dawoud, I. Taha, S.J. Ebeid, Journal of Manufacturing Processes 21 (2016) 39-45.

24. S.F. Costa, F.M. Duarte, J.A. Covas, Virtual and Physical Prototyping 10 (2014) 35-46.

25. R. Dell'Erba, et al., Polymer 42 (2001) 7831-7840.

26. C. Pouteau, et al., Comptes Rendus Biologies 327 (2004) 935-943.

27. J. Li, Y. He, Y. Inoue, Polymer International 52 (2003) 949-955

28. O. Gordobil, et al., Polymer Degradation and Stability 108 (2014) 330-338.

29. R. Surana, et al., Thermochimica Acta 433 (2005) 173-182.

30. J.M. Hutchinson, Progress in Polymer Science 20 (1995) 703-760.

31. C. Zhou, et al., Polymer 90 (2016) 111-121.

32. L. Baldenegro-Perez, et al., Polymers 6 (2014) 583-600.

33. M. Yasuniwa, et al., Journal of Polymer Science Part B: Polymer Physics 42 (2004) 25-32.

34. A. Melocchi, et al., International Journal of Pharmaceutics 509 (2016) 255-263.

35. S.I.G, S.C.D., M. Atif Yardimci, in: Solid Freeform Fabrication Symposium Proceedings, Austin, TX, 1997.

36. W. Wu, et al., Materials 8 (2015) 5834-5846.

37. Y.K. Chou, Y. Zhang, Proceedings of the Institution of Mechanical Engineers Part B: Journal of Engineering Manufacture 220 (2006) 1663-1671.

38. F. Awaja, et al., Progress in Polymer Science 34 (2009) 948-968. 